Case Report

\title{
Radiograms Obtained during Anterior Cervical Decompression and Fusion Can Mislead Surgeons into Performing Surgery at the Wrong Level
}

\author{
Chikato Mannoji,, Masao Koda, ${ }^{2}$ Takeo Furuya, ${ }^{2}$ Yuzuru Okamoto, ${ }^{1}$ Tamiyo Kon, \\ Kazuhisa Takahashi, ${ }^{2}$ Masashi Yamazaki, ${ }^{3}$ and Masazumi Murakami ${ }^{1}$ \\ ${ }^{1}$ Department of Orthopaedic Surgery, Chiba Aoba Municipal Hospital, Aobacho 1273-2, Chuo-ku, Chiba 260-0852, Japan \\ ${ }^{2}$ Department of Orthopaedic Surgery, Chiba University Graduate School of Medicine, 1-8-1 Inohana, Chuo-ku, Chiba 260-8677, Japan \\ ${ }^{3}$ Department of Orthopaedic Surgery, Graduate School of Comprehensive Human Sciences, University of Tsukuba, 1-1-1 Tennodai, \\ Tsukuba, Ibaraki 305-8575, Japan
}

Correspondence should be addressed to Chikato Mannoji; chikato.m@gmail.com

Received 11 June 2014; Revised 20 September 2014; Accepted 6 October 2014; Published 16 October 2014

Academic Editor: Georg Singer

Copyright (C) 2014 Chikato Mannoji et al. This is an open access article distributed under the Creative Commons Attribution License, which permits unrestricted use, distribution, and reproduction in any medium, provided the original work is properly cited.

\begin{abstract}
A 68-year-old woman who suffered from C5 nerve palsy because of a C4-5 disc herniation was referred to our hospital. We conducted anterior cervical decompression and fusion (ACDF) at the C4-5 level. An intraoperative radiogram obtained after exposure of the vertebrae showed that the level at which we were going to perform surgery was exactly at the C4-5 level. After bone grafting and temporary plating, another radiogram was obtained to verify the correct placement of the plate and screws, and it appeared to show that the plate bridged the C5 and C6 vertebrae at the incorrect level. The surgeon was astonished and was about to begin decompression of the upper level. However, carefully double-checking the level with a C-arm image intensifier before additional decompression verified that the surgery was conducted correctly at C4-5. Cautiously double-checking the level of surgery with a C-arm image intensifier is recommended when intraoperative radiograms suggest surgery at the wrong level.
\end{abstract}

\section{Introduction}

Wrong-site surgery (WSS) is rare [1-15], but once it occurs, it distresses both patients and doctors [6]. Therefore, spine surgeons should make every effort to avoid wrong-site surgery. Here, we report a rare experience where a radiogram, which was obtained during anterior cervical decompression and fusion (ACDF), almost misled a surgeon into performing surgery at the wrong level.

\section{Case Report}

A 68-year-old woman suffered from left-side C5 nerve palsy because of a C4-5 disc herniation. Manual muscle testing scores of her left-side deltoid and biceps were 1 and 4 , respectively, and physical examination showed no symptoms of myelopathy. Magnetic resonance imaging and computed tomography (CT) after myelography showed that the herniated disc at the C4-5 level compressed her left C5 nerve (Figure 1).

We conducted ACDF at the C4-5 level. During ACDF, we always obtain two radiograms to avoid WSS. One is taken after exposure of the vertebrae, with a needle inserted into a disc to verify that the level at which the decompression and fusion are to be conducted is correct. The other one is taken after temporary fixation of a plate following bone grafting to verify the correct placement of the plate and screws. During the surgery for the current case, the first radiogram showed that the needle was inserted into the C4-5 disc (Figure 2), so we continued the surgery and performed the herniotomy and bone grafting. After bone grafting, we positioned a plate to bridge the $\mathrm{C} 4$ and $\mathrm{C} 5$ vertebrae and fixed them temporarily. The radiogram after temporary placement of the plate astonished the surgeon because it appeared to show that the plate bridged the C5 and C6 vertebrae (Figure 3). The surgeon 


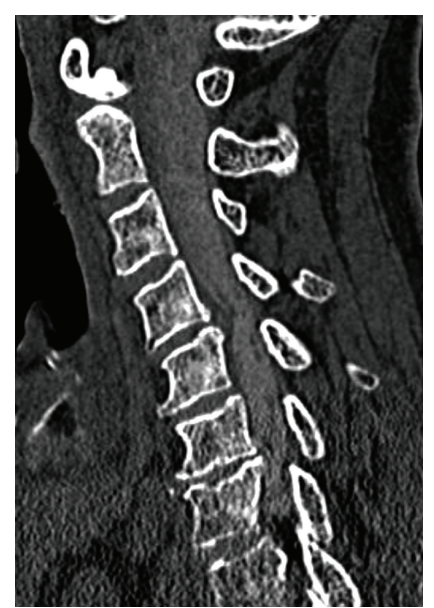

(a)

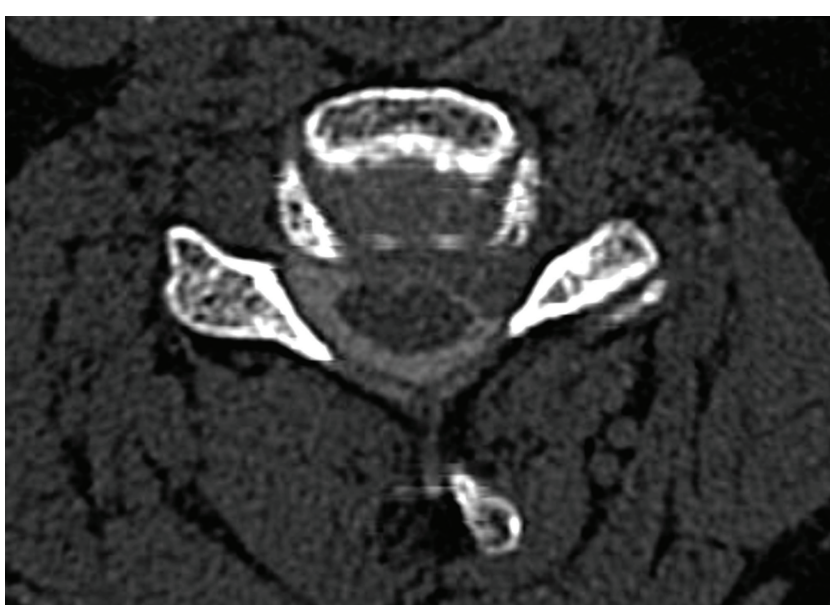

(b)

Figure 1: Computed tomography after myelography showing left-side C4-5 disc herniation. (a) Parasagittal view and (b) axial view at the C4-5 level.

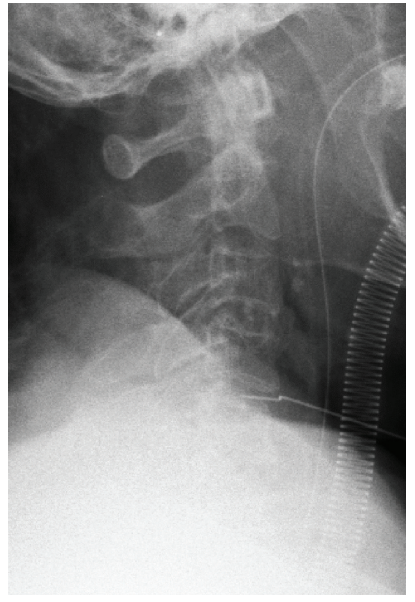

FIgURE 2: The first intraoperative radiogram after exposure of the vertebrae showing the needle inserted into the $\mathrm{C} 4-5$ disc.

removed the plate and was about to begin decompression of the upper level. However, because we were unable to determine the reason why the level was apparently incorrect, we decided to double-check the level with a C-arm image intensifier before decompression of the upper level. The image verified that the surgery was conducted correctly at the level of C4-5, and not C5-6 as we were mistakenly led to believe. The final radiograms before the extubation also verified that the surgery was correctly performed at the C4-5 level (Figure 4).

After completing the surgery, we investigated why the radiogram apparently indicated the wrong site. Using a 3D CT image obtained after the surgery, we were able to construct a picture in which it appeared as if the plate bridged the $\mathrm{C} 5$ and $\mathrm{C} 6$ vertebrae (Figure 5). This revealed that the radiogram was taken from a caudal to cranial perspective during the surgery, and that the direction of exposure was not perpendicular to the axis of the spine.

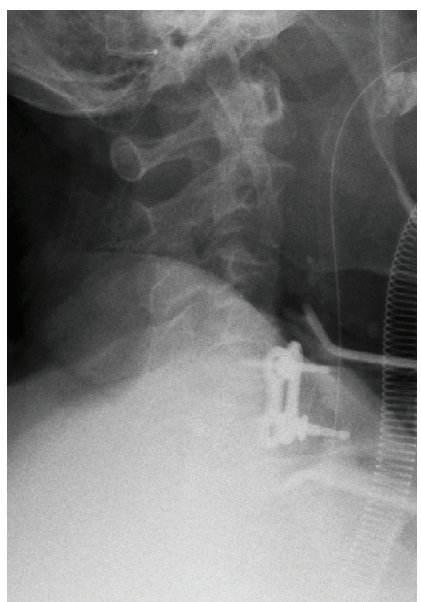

FIGURE 3: The second intraoperative radiogram after decompression, bone grafting and temporally plate fixation. It appears to show that the plate bridges the C5 and C6 vertebrae.

\section{Discussion}

Various risk factors of WSS of the spine have been reported including emergency surgery, obesity, anatomic variations, time pressure to complete surgery, unusual equipment, multiple surgeons involved in the surgery, multiple procedures in a single surgery, and insufficient communication between the surgical team and the patient $[10,12,14,16-20]$. In addition, failure to identify the vertebral level by intraoperative radiograms and misinterpretation of the radiogram are especially associated with wrong-level surgery $[18,20,21]$. As for cervical spine surgery, inadequate radiograms of the lower cervical spine hidden by the shoulders and cervical anomalies including Klippel-Feil syndrome and a block vertebra at C2-3 are major causes of wrong-level surgery [20]. In the current case, the patient did not have any of these factors. 


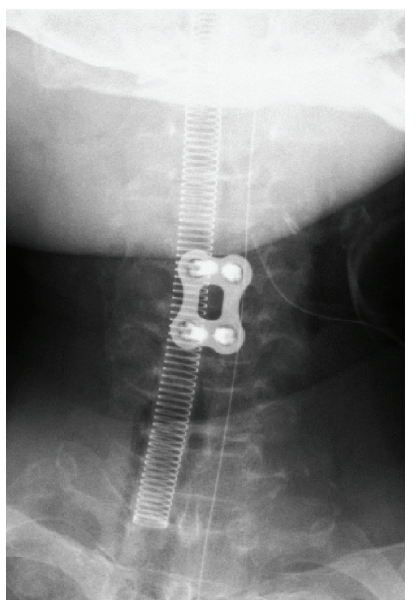

(a)

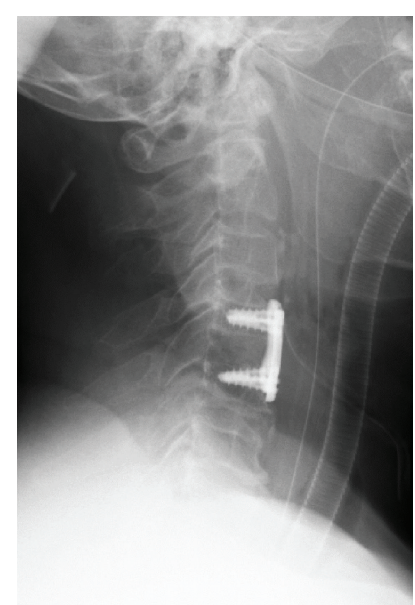

(b)

FIGURE 4: The final radiograms before extubation showed that ACDF was indeed performed at the correct level at C4-5. (a) Anteroposterior view and (b) lateral view.

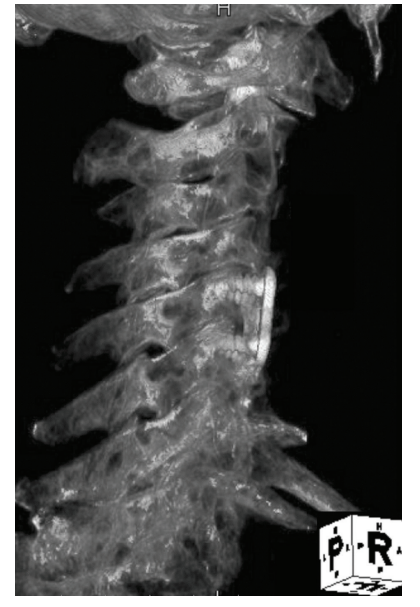

FIGURE 5: A constructed picture simulating the second intraoperative radiogram was obtained from $3 \mathrm{D} C \mathrm{CT}$ after the surgery. The plate appeared to bridge the C5 and C6 vertebrae.

There are some protocols for preventing WSS [22-24]. However, the effectiveness of the implementation of these protocols is controversial. Vachhani and Klopgenstein reported that the universal protocol (UP) by the Joint Commission on Accreditation of Healthcare Organizations was effective to reduce WSS events [25], but Wong and Watters III reported the UP was not effective [26]. Kwaan et al. reviewed cases and concluded that even the implementation of the UP would not have prevented $38 \%$ of WSS [8]. One of the main methods to avoid WSS is the use of radiograms during the surgery and this method is supported by many surgeons $[6,7,14,15,27-29]$. However, radiograms during the surgery cannot avoid every case of WSS because some patients have congenital anomaly of the spine or where radiograms are inadequate $[10,12,25]$. Some authors recommend using fluoroscopy during the surgery to identify correct levels for spinal surgery [13, 15, 30, 31]. Mayer et al. reported that surgeons now use fluoroscopy more frequently than plain radiograms during posterior surgery of the thoracic and lumbar spine, and surgeons who experienced WSS tend to have used plain radiograms more than fluoroscopy [31]. Intraoperative CT scan [32-36] is also useful to prevent WSS, but using this method routinely for only localizing the correct level is not practical.

In the current case, we obtained two radiograms during ACDF and the second radiogram almost misled a surgeon into performing unnecessary decompression at the wrong level, even though the patient did not have any anatomical anomalies of the cervical spine and the shoulders of the patient were pulled caudally during the radiograms to make it easier to see the correct level. On the other hand, a C-arm image intensifier clearly showed that we performed ACDF at the correct level. The cause of this event was that the second radiogram was inadequate and the surgeon could therefore not correctly interpret the picture. We constructed another picture from the 3D CT after the surgery that was similar to the second radiogram. This constructed picture revealed that the second radiogram was taken from a caudal to cranial perspective and the direction of exposure was not perpendicular to the axis of the spine as believed. Careful examination of Figure 5 shows that the "C3-4" disc is not clearly visualized. However, the surgeon in the operating room is under pressure to interpret radiograms quickly in less than ideal conditions and so their evaluation is compromised if they are inadequate. We highly recommend using a Carm image intensifier to double-check the level of surgery if an intraoperative radiogram shows an unexpected finding, because a $\mathrm{C}$-arm image intensifier can provide many images on many planes at once whereas plain radiograms do not offer real time feedback when the image is oblique or obscured by the shoulders. The fact that surgeons now use fluoroscopy more frequently than plain radiograms and surgeons who experienced WSS tend to have used plain radiograms more than fluoroscopy [31] also indicates that fluoroscopy is more useful than plain radiograms. 
In conclusion, radiograms obtained during ACDF surgery can mislead surgeons into performing surgery at the wrong site. Cautiously double-checking the surgical level with a C-arm image intensifier is recommended when intraoperative radiograms suggest wrong-site surgery.

\section{Conflict of Interests}

The authors declare that there is no conflict of interests regarding the publication of this paper.

\section{References}

[1] R. W. Williams, "Microlumbar discectomy. A conservative surgical approach to the virgin herniated lumbar disc," Spine, vol. 3, no. 2, pp. 175-182, 1978.

[2] N. Eie, T. Solgaard, and H. Kleppe, "The knee-elbow position in lumbar disc surgery: a review of complications," Spine, vol. 8, no. 8, pp. 897-900, 1983.

[3] F. Ruggieri, L. Specchia, S. Sabalat, G. Galli, P. Ruggieri, and S. Arlecchini, "Lumbar disc herniation: diagnosis, surgical treatment, recurrence: a review of 872 operated cases," Italian Journal of Orthopaedics and Traumatology, vol. 14, no. 1, pp. 1522, 1988.

[4] N. A. Ebraheim, C. Inzerillo, and R. Xu, "Are anatomic landmarks reliable in determination of fusion level in posterolateral lumbar fusion?” Spine, vol. 24, no. 10, pp. 973-974, 1999.

[5] E. G. Meinberg and P. J. Stern, "Incidence of wrong-site surgery among hand surgeons," Journal of Bone and Joint Surgery, vol. 85, no. 2, pp. 193-197, 2003.

[6] R. Goodkin and L. L. Laska, "Wrong disc space level surgery: medicolegal implications," Surgical Neurology, vol. 61, no. 4, pp. 323-342, 2004.

[7] J. M. Ammerman, M. D. Ammerman, J. Dambrosia, and B. J. Ammerman, "A prospective evaluation of the role for intraoperative $\mathrm{x}$-ray in lumbar discectomy. Predictors of incorrect level exposure," Surgical Neurology, vol. 66, no. 5, pp. 470-473, 2006.

[8] M. R. Kwaan, D. M. Studdert, M. J. Zinner et al., "Incidence, patterns, and prevention of wrong-site surgery," Archives of Surgery, vol. 141, no. 4, pp. 353-358, 2006.

[9] S. C. Seiden and P. Barach, "Wrong-side/wrong-site, wrongprocedure, and wrong-patient adverse events: are they preventable?" Archives of Surgery, vol. 141, no. 9, pp. 931-939, 2006.

[10] B. S. Jhawar, D. Mitsis, and N. Duggal, "Wrong-sided and wrong-level neurosurgery: a national survey," Journal of Neurosurgery: Spine, vol. 7, no. 5, pp. 467-472, 2007.

[11] J. R. Clarke, J. Johnston, and E. D. Finley, "Getting surgery right," Annals of Surgery, vol. 246, no. 3, pp. 395-403, 2007.

[12] M. G. Mody, A. Nourbakhsh, D. L. Stahl, M. Gibbs, M. Alfawareh, and K. J. Garges, "The prevalence of wrong level surgery among spine surgeons," Spine, vol. 33, no. 2, pp. 194-198, 2008.

[13] J.-L. Pao, W.-C. Chen, and P.-Q. Chen, "Clinical outcomes of microendoscopic decompressive laminotomy for degenerative lumbar spinal stenosis," European Spine Journal, vol. 18, no. 5, pp. 672-678, 2009.

[14] J. Devine, N. Chutkan, D. C. Norvell, and J. R. Dettori, "Avoiding wrong site surgery: a systematic review," Spine, vol. 35, no. 9, pp. S28-S36, 2010.

[15] C. Irace and C. Corona, "How to avoid wrong-level and wrongside errors in lumbar microdiscectomy: clinical article," Journal of Neurosurgery: Spine, vol. 12, no. 6, pp. 660-665, 2010.
[16] H. C. Pheasant, "Sources of failure in laminectomies," The Orthopedic Clinics of North America, vol. 6, no. 1, pp. 319-329, 1975.

[17] J. M. Ammerman and M. D. Ammerman, "Wrong-sided surgery," Journal of Neurosurgery: Spine, vol. 9, no. 1, pp. 105-106, 2008.

[18] M. A. James, J. G. Seiler III, J. J. Harrast, S. E. Emery, and S. Hurwitz, "The occurrence of wrong-site surgery self-reported by candidates for certification by the American Board of Orthopaedic Surgery," Journal of Bone and Joint Surgery A, vol. 94, no. 1, p. e2, 2012.

[19] M. W. Groff, J. E. Heller, E. A. Potts, P. V. Mummaneni, C. I. Shaffrey, and J. S. Smith, "A survey-based study of wronglevel lumbar spine surgery: the scope of the problem and current practices in place to help avoid these errors," World Neurosurgery, vol. 79, no. 3-4, pp. 585-592, 2013.

[20] M. A. Palumbo, A. J. Bianco, S. Esmende, and A. H. Daniels, "Wrong-site spine surgery," Journal of the American Academy of Orthopaedic Surgeons, vol. 21, no. 5, pp. 312-320, 2013.

[21] C. A. Fager, "Malpractice issues in neurological surgery," Surgical Neurology, vol. 65, no. 4, pp. 416-421, 2006.

[22] American Academy of Orthopedic Surgeons, Information Statement WRONG-SITE SURGERY, American Academy of Orthopedic Surgeons, Rosemont, Ill, USA, 1997, http://www.aaos.org/ about/papers/advistmt/1015.asp.

[23] North American Spine Society, Sign, Mark \& X-ray (SMaX): prevent Wrong-Site Surgery, North American Spine Society, La Grange, Ill, USA, 2014, http://www.spineline.org/Pages/ PracticePolicy/ClinicalCare/SMAX/Default.aspx.

[24] Joint Commission on Accreditation of Healthcare Organizations, Universal Protocol for Preventing Wrong Site, Wrong Procedure, Wrong Person Surgery, Joint Commission on Accreditation of Healthcare Organizations, Oakbrook Terrace, Ill, USA, 2003, http://www.jointcommission.org/standards_information/up.aspx.

[25] J. A. Vachhani and J. D. Klopgenstein, "Incidence of neurosurgical wrong-site surgery before and after implementation of the universal protocol," Neurosurgery, vol. 72, no. 4, pp. 590-595, 2013.

[26] D. A. Wong and W. C. Watters III, “To err is human: quality and safety issues in spine care," Spine, vol. 32, no. 11, pp. S2-S8, 2007.

[27] R. K. Michaels, M. A. Makary, Y. Dahab et al., "Achieving the national quality forum's "never events": prevention of wrong site, wrong procedure, and wrong patient operations," Annals of Surgery, vol. 245, no. 4, pp. 526-532, 2007.

[28] U. G. Longo, M. Loppini, G. Romeo, N. Maffulli, and V. Denaro, "Errors of level in spinal surgery: an evidence-based systematic review," Journal of Bone and Joint Surgery-Series B, vol. 94, no. 11, pp. 1546-1550, 2012.

[29] W. Hsu, R. M. Kretzer, M. J. Dorsi, and Z. L. Gokaslan, "Strategies to avoid wrong-site surgery during spinal procedures," Neurosurgical Focus, vol. 31, no. 4, article E5, 2011.

[30] H. Singh, S. A. Meyer, A. C. Hecht, and A. L. Jenkins, "Novel fluoroscopic technique for localization at cervicothoracic levels," Journal of Spinal Disorders and Techniques, vol. 22, no. 8, pp. 615-618, 2009.

[31] J. Mayer, R. P. Dnag, G. F. D. Prieto, S. Qureshi, S. K. Cho, and A. Hecht, "Analysis of the techniques for thoracic and lumbar level localization during posterior spine surgery and the occurrence of wrong level surgery: results from a National survey," The Spine Journal, vol. 12, no. 9, pp. S71-S72, 2012. 
[32] A. C. Hecht, S. M. Koehler, J. C. Laudone, A. Jenkins, and S. Qureshi, "Is intraoperative CT of posterior cervical spine instrumentation cost-effective and does it reduce complications?" Clinical Orthopaedics and Related Research, vol. 469, no. 4, pp. 1035-1041, 2011.

[33] F. Costa, A. Cardia, A. Ortolina, G. Fabio, A. Zerbi, and M. Fornari, "Spinal navigation: standard preoperative versus intraoperative computed tomography data set acquisition for computer-guidance system: radiological and clinical study in 100 consecutive patients," Spine, vol. 36, no. 24, pp. 2094-2098, 2011.

[34] E. Ughwanogho, N. M. Patel, K. D. Baldwin, N. R. Sampson, and J. M. Flynn, “Computed tomography-guided navigation of thoracic pedicle screws for adolescent idiopathic scoliosis results in more accurate placement and less screw removal," Spine, vol. 37, no. 8, pp. E473-E478, 2012.

[35] G. Cui, Y. Wang, T.-H. Kao et al., "Application of intraoperative computed tomography with or without navigation system in surgical correction of spinal deformity: a preliminary result of 59 consecutive human cases," Spine, vol. 37, no. 10, pp. 891-900, 2012.

[36] J. K. Houten, R. Nasser, and N. Baxi, "Clinical assessment of percutaneous lumbar pedicle screw placement using the $\mathrm{O}$-arm multidimensional surgical imaging system," Neurosurgery, vol. 70, no. 4, pp. 990-995, 2012. 


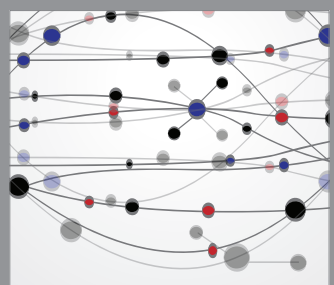

The Scientific World Journal
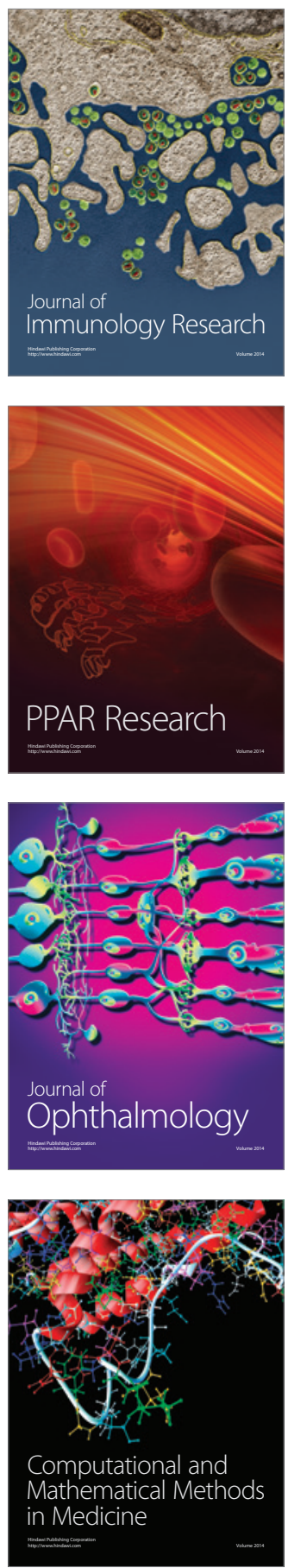

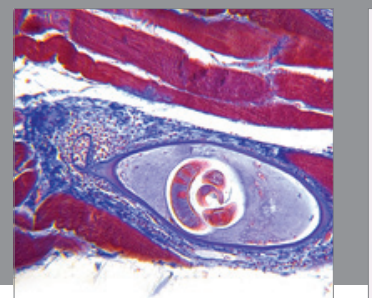

Gastroenterology

Research and Practice
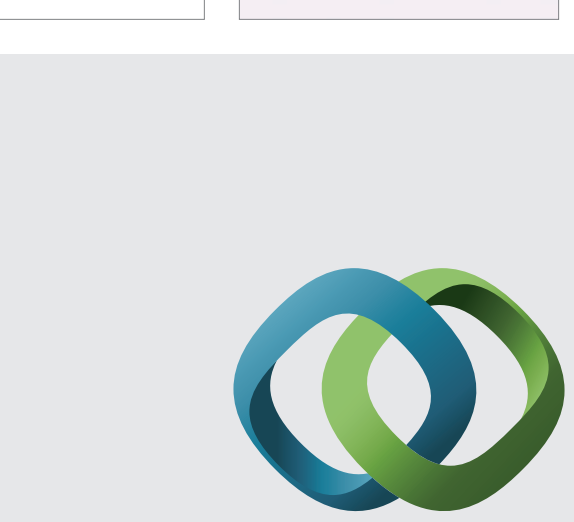

\section{Hindawi}

Submit your manuscripts at

http://www.hindawi.com
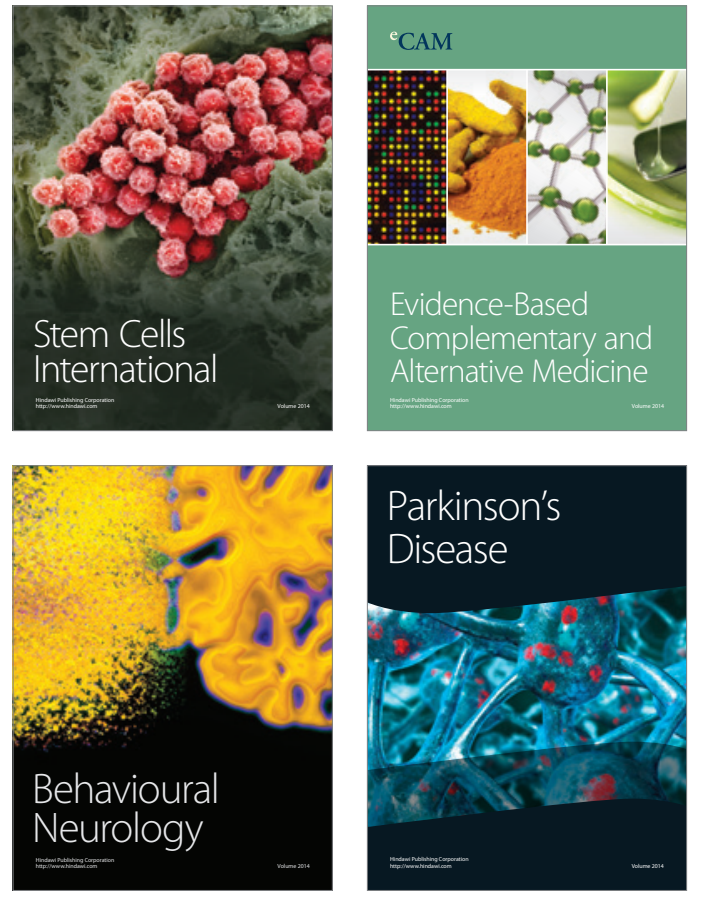
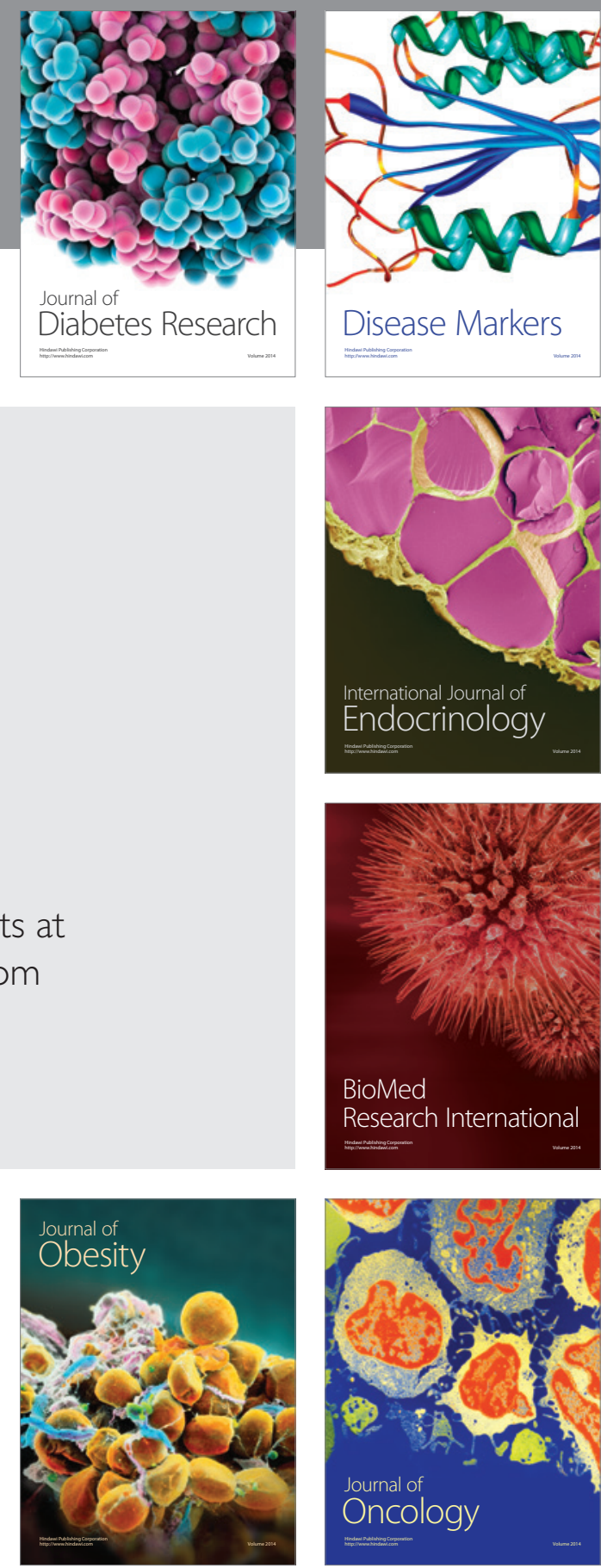

Disease Markers
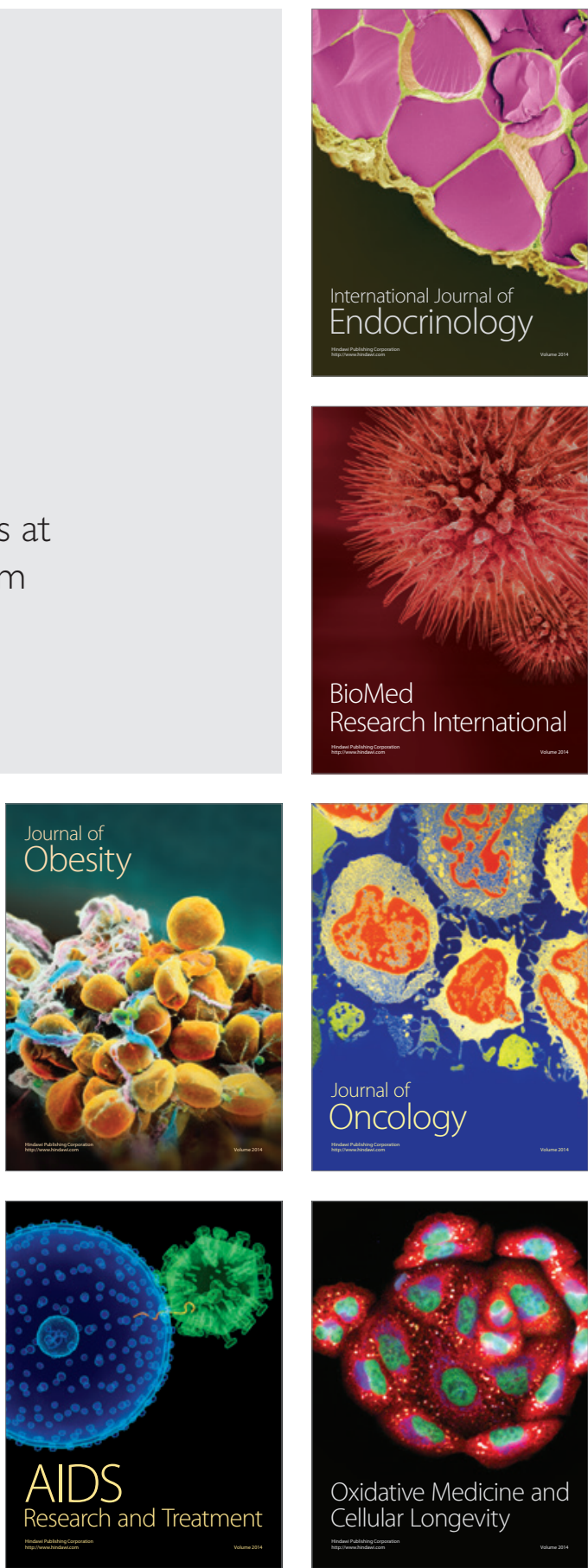